\title{
Efecto de la utilización de pulseras inteligentes para el incremento de la actividad física en adolescentes de un entorno rural: Estudio Piloto
}

\author{
Effect of the Use of Smart Bracelets to Increase Physical \\ Activity in Adolescents in a Rural Environment: Pilot Study
}

Gema Díaz-Quesada' (웅

Esther Puga-González ${ }^{10}$

Inés M. Muñoz-Galiano ${ }^{*}$ *(1)

1. Facultad de Humanidades y Ciencias de la Educación, Universidad de Jaén, España.

\begin{abstract}
Resumen
Los niveles de actividad física (AF) cada vez son más bajos. Las recomendaciones de 60 minutos de actividad física moderada-vigorosa (AFMV) y entre 10.000 y 16.000 pasos al día para niños y adolescentes, solo son cumplidas por grupos minoritarios. Los programas de intervención utilizando dispositivos inteligentes son una buena opción para el incremento de la AF. El objetivo de este estudio fue evaluar el efecto de la utilización de pulseras inteligentes para el incremento de AF en una muestra de un entorno rural. Un total de ocho estudiantes de entre 14 y 16 años participaron en este estudio. Los sujetos portaron una pulsera de actividad física Xiaomi Mi Band 4 durante dos semanas, donde se registró el número de pasos y los minutos de AFMV. La primera Semana de Control (S1), fue una semana de rutina escolar habitual. La segunda Semana de Intervención (S2), fue una intervención de promoción de la AF orientada al incremento de la misma por medio de retos. Los sujetos mostraron en la primera semana una media de 7.200 pasos/día y 173 minutos/día y, durante la segunda, una media de 12.300 pasos/día y 212 minutos/día de AFMV, mostrando así un aumento importante durante la segunda semana, respecto a la primera semana. Diferentes estudios muestran grandes incrementos de AFMV al aplicar programas de intervención, consiguiendo así que se cumplan las recomendaciones mínimas diarias de minutos de AFMV y de pasos. Se evidencia que las pulseras de AF influyen de manera positiva para el aumento de los niveles de AF, además de resultar un instrumento motivante para el alumnado.
\end{abstract}

Palabras clave: Salud, alumnado, educación, intervención, pasos, recomendaciones

\begin{abstract}
Physical activity (PA) levels are getting lower and lower. Recommendations of 60 minutes of moderate-to-severe physical activity (MVPA) and 10,000-16,000 steps per day for children and adolescents are only met by minority groups. Intervention programs using smart devices are a good option for increasing PA. The aim of this study was to evaluate the effect of using smart devices to increase PA in a rural setting sample. A total of eight students aged 14-16 years participated in this study. Subjects wore a Xiaomi Mi Band 4 physical activity bracelet for two weeks, where the number of steps and minutes of MVPA were recorded. The first Control Week (CW) was a week of the regular school routine. The second Intervention Week (IW) was a PA promotion intervention to increase it through challenges. The subjects showed an average of 7200 steps/day and 173 minutes/day during the first week and an average of 12300 steps/day and 212 minutes/day of MVPA during the second week, showing a significant increase during the second week, compared to the first week. Different studies show large increases of MVPA when applying for intervention programs, thus achieving the minimum daily recommendations of MVPA minutes and steps. It is found that PA bracelets positively influence PA intervention programs, and be a motivating instrument for students.
\end{abstract}

Keywords: Health, students, education, intervention, steps, recommendations.

\section{* Autor de correspondencia: Inés M. Muñoz-Galiano, imunoz@ujaen.es}

Recibido: 02 de noviembre de 2020

Aceptado: 02 de diciembre de 2020

Publicado: 31 de enero de 2021

Como citar (APA): Díaz-Quesada, G., Puga-González, E., \& Muñoz-Galiano, I. (2021). Efecto de la utilización de pulseras inteligentes para el incremento de la actividad física en adolescentes de un entorno rural: Estudio Piloto. JUMP, (3), 10-16. https://doi.org/10.17561/jump.n3.2 


\section{Introducción}

Actualmente diferentes investigaciones muestran que cada vez son más bajos los niveles de actividad física (AF) en la población mundial, durante la infancia y la adolescencia nos encontramos con dos medidas para calcular la AF (Andersen, Mota \& Di Pietro, 2016; Tremblay et al., 2017). Por un lado, distintos autores y organismos establecen las recomendaciones de realizar 60 minutos diarios de actividad física moderada-vigorosa (AFMV), aconsejándose que, de los siete días de la semana, al menos tres días incluyan actividades que impliquen una actividad física vigorosa (AFV) (Center for Disease Control and Prevention [CDC], 2011; U.S. Department of Health and Human Services, 2018; World Health Organization [WHO], 2010). Por otro lado, diversas investigaciones establecen las recomendaciones de realizar entre 10000 y 16000 pasos diarios (Tudor-Locke et al., 2009; Tudor-Locke et al., 2011).

Existen grupos mayoritarios que no están cumpliendo dichas recomendaciones, (Katzmarzyk et al., 2016; Wijtzes et al., 2016; WHO, 2014). Según estudios recientes más del $80 \%$ de los adolescentes no llegan a los niveles mínimos recomendados (Guthol et al., 2019).

Debido a ello, actualmente en el mercado se puedenencontrar una gran cantidad de dispositivos portátiles validados que miden las actividades relacionadas con la salud con la finalidad de promover la AF (Lee, Kim \& Welk, 2014; Price et al., 2017). Actualmente los dispositivos más precisos que se encuentran en el mercado son los acelerómetros, pero el costo es relativamente alto y requiere un procesamiento de datos complejo, por lo que en ciertas investigaciones pueden no resultar prácticos o fácilmente asequibles (Keadle et al., 2014; Migueles et al., 2017). A pesar de ello, hay otros dispositivos basados en acelerómetros como pulseras o podómetros que también miden la $A F$, siendo más pequeños, fáciles de utilizar y ofreciendo precios más asequibles (Bai et al., 2016; Sperlich \& Holmberg, 2017). Por lo tanto, dichos dispositivos podrían ser una estrategia efectiva para la promoción de la AF de una forma atractiva y lúdica (Evenson, Goto \& Furberg, 2015; Ridgers, McNarry \& Mackintosh, 2016), estableciendo objetivos específicos a corto plazo para la consecución de un mejor rendimiento (Pearson, 2012).
Por este motivo, cada vez hay más estudios con estrategias de intervención para el incremento de la AF en escolares usando diferentes dispositivos de recolección de datos (Böhm et al., 2019; Kriemler et al., 2011). El centro escolar toma un papel muy importante, pues los escolares pasan aproximadamente un tercio del día en el colegio (Gauthier et al., 2012) y según el Ministerio de Educación Británico el entorno escolar debe proporcionar al menos la realización de la mitad de tiempo recomendado de AFMV diaria (BC Ministry of Education, 2011). Esto, unido a que las familias están saturadas con los horarios laborales y las responsabilidades del hogar, hacen que el tiempo que pueden dedicar a sus hijos para incrementar dichos niveles sea escaso (Banga et al., 2020) y convierte al centro escolar en el lugar idóneo para promover la realización de AF en niños y jóvenes, además de contribuir a la consecución de las recomendaciones diarias (Heath et al., 2012; Hills, Dengel \& Lubans, 2015; Martínez-Gómez et al., 2013). Algunos autores muestran programas de intervención para aumentar la AF diseñados y programados para entornos escolares en diferentes grupos de edad (Hayes \& Van Camp, 2015; Pittman, 2018).

Por lo tanto, el objetivo de este estudio fue evaluar el efecto de la utilización de pulseras inteligentes para el incremento de AF en una muestra de un entorno rural.

\section{Material y Método}

\section{Muestra}

Se seleccionaron un total de 8 escolares de tercero de la E.S.O. (14,5 \pm 0,50 años). La selección de la muestra se realizó a través de un método de muestreo intencional de acuerdo con los criterios de accesibilidad y proximidad (Otzen \& Manterola, 2017). Todos ellos pertenecían a un entorno rural (<10.000 habitantes) (Chillón et al., 2011), y sus tutores firmaron un consentimiento por escrito para participar en el estudio (JUN.17/6).

\section{Procedimiento}

El estudio se llevó a cabo en dos partes: Semana de Control (S1) consistente en una semana de rutina normal y Semana de Intervención (S2) para la promoción de la AF. Para la S2 se indicó que se incrementase el $20 \%$ de $A F$, respecto a los valores que, individualmente cada uno de los sujetos obtuvo en la S1. En ambas semanas los 
participantes portaron en la muñeca la pulsera de AF Xiaomi Mi band 4 (Anhui Huami Information Technology Co., Ltd., China) en todo momento, incluida la ducha y dormir. La pulsera es portátil y cómoda de portar, además de ser impermeable y poder sumergirse hasta 50 metros. El dispositivo registra la AF contabilizando el volumen de pasos y los minutos de AFMV. El dispositivo dispone de una aplicación para móvil o Tablet (Mi Fit, Anhui Huami Information Technology Co., Ltd., China) para una mejor visualización de los datos. Este dispositivo ha demostrado ser preciso al evaluar la AF y los pasos (El-Amrawy \& Nounou, 2015; Wang et al., 2017). Se le dio al alumnado unas directrices para la S2, durante la mañana se les animó a ir y volver al instituto andado, realizar AF durante el recreo, entre clase y clase, y bajar al patio para dar una vuelta; por la tarde se les instó para que las acciones que tuvieran que realizar fuera de casa las hiciesen caminando. Los participantes, a su vez, disponían de un diario para registrar los datos diariamente. Se realizó un análisis del total de pasos/día y AFMV en minutos/día de los siguientes periodos: Días de la semana por separado, Total de la Semana (Lunes a Domingo), Días entre semana y Días en fin de semana.

\section{Análisis estadístico}

Todos los análisis estadísticos se realizaron empleando el paquete estadístico IBM SPSS Statistics para Windows en su versión 25.0 (IBM Corp. Released 2017. IBM SPSS Statistics for Windows, Version 25.0. Armonk, NY: IBM Corp.), y el paquete estadístico (JAMOVI) en su versión 1.2 (https://www.jamovi.org). Se realizó un análisis descriptivo de los datos como media y desviación típica. En segundo lugar, se empleó el test de normalidad Shapiro-Wilk, que confirmó una distribución normal. En tercer lugar, se empleó el t-test para muestras pareadas Student's para ver las diferencias entre semana (Semana 1 versus Semana 2). El nivel de significación se fijó a $p \leq$ 0,05 para las diferentes pruebas.

\section{Resultados}

En la Tabla 1 se muestran las diferencias en cuanto a pasos y nivel de AF en la S1 y S2.

Los resultados muestran un incremento de la AF en la semana 2 con un incremento del volumen de pasos todos los días de la semana. De igual forma este incremento se observa en el volumen de minutos de AFMV exceptuando el día sábado. Se observan diferencias estadísticamente significativas, con un volumen de pasos diarios más elevado en la semana 2 respecto a la semana 1 en los días: lunes $(p<0.05)$, martes y jueves $(p<0.01)$. Respecto a la cantidad de minutos de AFMV, se muestran diferencias estadísticamente significativas con valores más altos en la semana 2 respecto a la semana 1 solo en el día jueves $(p<0.01)$.

En la Tabla 2 se muestran las diferencias en cuanto a pasos y nivel de AF entre semana (Lunes a Viernes), Fin de Semana y Total de la Semana, a lo largo de las dos semana evaluadas.

Como se puede observar en los resultados se aprecian diferencias estadísticamente significativas en los pasos Entre Semana, los pasos en el Total de la Semana, los minutos Entre Semana y los minutos en el Total de la Semana, siendo los valores más altos en la semana 2 respecto a la semana $1(p \leq 0,05)$.

\section{Discusión}

Este trabajo evalúa el efecto de la utilización de pulseras inteligentes para el incremento de AF en una muestra de un entorno rural a lo largo de una S1 y S2. Los principales hallazgos de este estudio muestran como en la población seleccionada, no se cumplían las recomendaciones diarias de AFMV ni pasos en una semana de rutina normal S1. También se puede apreciar cómo unas directrices sobre la promoción de la AF, han ayudado a que se produzca un aumento importante en los niveles de AF en la S2, respecto a la S1. A su vez, dicho incremento ha supuesto que esta población cumpla con las recomendaciones diarias en volumen de pasos o de minutos de AFMV, las cuales no se cumplían durante la S1.

Es destacable que los escolares no cumplían con las recomendaciones diarias de AFMV ni de pasos durante la S1, esto coincide con otros estudios en los que en una semana de rutina normal tampoco se cumplen dichas recomendaciones (Miguel-Berges et al., 2019; Wijtzes et al., 2016). Dándose esta misma situación tanto en edades más tempranas (Santaliestra-Pasías et al., 2018; Wyszyńska et al., 2020), como en edades más avanzadas donde ya existe una mayor concienciación y madurez (Guthol et al., 2019; Mayorga-Vega et al., 2019). Esto resultados ponen de relieve la necesidad de establecer estrategias e intervenciones para cambiar dichos resultados (Hardy et al., 2008; Zongo et al., 2017). 
Tabla 1. Diferencias en la actividad física desarrollada durante cada día de la semana, S1 versus S2

\begin{tabular}{lccccc}
\hline & S1 & S2 & $\boldsymbol{p}$ & Cohen's & Cambio (\%) \\
\hline Lunes (pasos/día) & $7419,25 \pm 4968,20$ & $13218,00 \pm 8137,72$ & 0,042 & $-0,88$ & +78 \\
Martes (pasos/día) & $7209,75 \pm 3079,98$ & $15993,63 \pm 3772,28$ & 0,005 & $-1,43$ & +122 \\
Miércoles (pasos/día) & $8664,13 \pm 7717,33$ & $12696,38 \pm 5659,60$ & 0,228 & $-0,47$ & +47 \\
Jueves (pasos/día) & $5636,50 \pm 4275,57$ & $12344,38 \pm 2365,22$ & 0,002 & $-1,68$ & +119 \\
Viernes (pasos/día) & $10315,13 \pm 7198,56$ & $1261,13 \pm 5300,21$ & 0,438 & $-0,29$ & +22 \\
Sábado (pasos/día) & $8243,38 \pm 7602,74$ & $10897,38 \pm 4200,44$ & 0,042 & $-0,88$ & +32 \\
Domingo (pasos/día) & $3287,63 \pm 2307,66$ & $8466,13 \pm 6460,37$ & 0,005 & $-1,43$ & +158 \\
Lunes AFMV (min/día) & $183,88 \pm 117,72$ & $206,50 \pm 93,42$ & 0,546 & $-0,22$ & +12 \\
Martes AFMV (min/día) & $190,75 \pm 91,88$ & $263,13 \pm 92,66$ & 0,216 & $-0,48$ & +38 \\
Miércoles AFMV (min/día) & $212,63 \pm 93,23$ & $215,88 \pm 112,12$ & 0,948 & $-0,02$ & +2 \\
Jueves AFMV (min/día) & $138,50 \pm 92,62$ & $260,13 \pm 53,78$ & 0,003 & $-1,57$ & +88 \\
Viernes AFMV (min/día) & $204,88 \pm 121,42$ & $260,75 \pm 175,44$ & 0,374 & $-0,34$ & +27 \\
Sábado AFMV (min/día) & $200,50 \pm 133,51$ & $170,13 \pm 79,03$ & 0,546 & $-0,22$ & -15 \\
Domingo AFMV (min/día) & $82,50 \pm 33,34$ & $111,50 \pm 59,18$ & 0,216 & $-0,48$ & +35 \\
\hline
\end{tabular}

AFMV: Actividad Física Moderada Vigorosa; M: Media; min: Minutos; p: significación; SD: Desviación típica

Tabla 2. Diferencias en la actividad física desarrollada Entre Semana, Fin de Semana y Total de la Semana. S1 versus S2

\begin{tabular}{|c|c|c|c|c|c|}
\hline & S1 & S2 & $p$ & Cohen's & Cambio (\%) \\
\hline Entre Semana (pasos/día) & $7848,95 \pm 4182,89$ & $13372,90 \pm 2910,90$ & 0,009 & $-1,27$ & +70 \\
\hline Fin de Semana (pasos/día) & $5765,50 \pm 4209,20$ & $9681,75 \pm 4300,98$ & 0,114 & $-0,64$ & +68 \\
\hline Total Semana (pasos/día) & $7253,68 \pm 4003,53$ & $12318,29 \pm 2237,68$ & 0,010 & $-1,25$ & +70 \\
\hline Entre Semana AFMV (min/día) & $140,26 \pm 62,84$ & $241,28 \pm 56,96$ & 0,002 & $-1,69$ & +72 \\
\hline Fin de Semana AFMV (min/día) & $141,50 \pm 70,63$ & $140,81 \pm 58,34$ & 0,982 & 0,01 & $-0,5$ \\
\hline Total Semana AFMV (min/día) & $173,38 \pm 74,79$ & $212,57 \pm 43,43$ & 0,038 & $-0,90$ & +23 \\
\hline
\end{tabular}

AFMV: Actividad Física Moderada Vigorosa; M: Media; min: Minutos; p: significación; SD: Desviación típica

Resulta interesante hacer hincapié en que esta problemática está sucediendo de forma general en toda la población, pudiendo tener el país de residencia una influencia sobre los niveles de AF (Beets et al., 2010; Van Tuyckom \& Scheerder, 2010). Es por ello, que resulta muy importante la investigación intercultural, para conocer los diversos contextos culturales e incluso conocer los tramos horarios del día durante los cuales tiene lugar la AF o la ausencia de la misma (Garriguet \& Colley, 2012).

Respecto a la intervención que se desarrolló para la promoción de AF durante la S2, cabe destacar la eficacia del mismo, pues se produjeron incrementos importantes respecto a la cantidad de minutos de AFMV y de los pasos diarios, estando estos resultados acordes con otros estudios en los que la intervención se desarrolló también con una S1 y una S2 (Gaudet, Gallant \& Bélanger, 2017), a su vez, este incremento también puede observarse en otros estudios en los que solo hubo semanas de intervención y en los que el alumnado incrementó los minutos de AFMV y de los pasos durante la primera semana y más notablemente hacia el fin de la intervención (Galy, Yacef \& Caillaud, 2019). Diversos autores combinan estos programas de intervención con otro componentemotivantecomo pueden ser las plataformas webs o mensajes de ánimo (Drehlich et al., 2020), obteniendo también incrementos importantes en los minutos de AFMV y los pasos diarios realizados.

En este estudio y en los analizados anteriormente, se observa que el aumento de minutos de AFMV y de los pasos diarios durante las intervenciones lograron que el alumnado cumpliera con las recomendaciones diarias y se muestra cómo el alumnado que no alcanza dichas recomendaciones, sí se acerca al cumplimiento de las mismas.

A pesar de ello, también existen estudios que a pesar de realizar algún programa de intervención no encontraron incrementos de minutos de AFMV y de los pasos diarios, de hecho, en algunos incluso se produjeron descensos (Kim et al., 2018; Nanney, 
2014; Rote, 2016). Hay que tener en cuenta que estos programas de intervención se realizaron con población universitaria.

Estos resultados muestran la importancia de planificar estrategias para el fomento de la AF, aunque cada estrategia tenga unas características diferentes, utilizando el uso de pulseras inteligentes en la realización de dichas intervenciones como un elemento motivante para la población adolescente (Brickwood et al., 2019; Bronikowski, Bronikowska, Maciaszek, \& Glapa, 2018).

Este trabajo no está exento de limitaciones, entre la que se destaca el tamaño de la muestra y el entorno rural del mismo.

\section{Conclusiones}

Existe evidencia de que las pulseras de AF pueden influir de manera positiva en los niveles de AF, además de resultar un instrumento motivante para el alumnado. Gracias a la creación de estas intervenciones, los adolescentes pueden llegar al cumplimiento diario recomendado de minutos de AFMV y de pasos.

\section{Aplicaciones prácticas}

Los resultados obtenidos en este estudio deben servir para incentivar cada vez más la creación de programas de intervención dentro del entorno escolar que ayuden a mejorar los niveles de AF. Estos programas a su vez, pueden utilizarse para crear hábitos de vida saludable y conseguir que las mejoras en dichos niveles se prolonguen a lo largo del tiempo. Además, se pueden combinar dichos programas con otras variables como el uso de páginas web donde mostrar sus logros, creación de asambleas o proyectos para explicar al resto de la comunidad educativa los beneficios que tienen estos nuevos hábitos saludables.

Un ejemplo de aplicación práctica sería que los responsables de los centros escolares tras realizar una revisión de la bibliografía existente sobre este tipo de intervencionesy los beneficios derivados de las mismas, capacitarán al centro de dispositivos inteligentes y crearán protocolos y programas de intervención enfocados al aumento de la AF, para que el profesorado pudiera llevarlo a cabo en el aula. De este modo, esto ayudaría a la creación de programas de vida activa, de desplazamientos activos, lecciones activas, etc.

\section{Financiación}

Este proyecto se lleva a cabo gracias al proyecto de innovación docente, PID PASEO (PIMED50_201921) de la Universidad de Jaén.

\section{Referencias}

Andersen, L. B., Mota, J., \& Di Pietro, L. (2016). Update on the global pandemic of physical inactivity. The Lancet, 388, 1255-1256. https://doi.org/10.1016/S0140-6736(16)30960-6

Banga, Y., Azhar, A., Sandhu, H., \& Tang, T. S. (2020). Dance "Cultural" Revolution: Tailoring a Physical Activity Intervention for South Asian Children. Journal of Immigrant and Minority Health, 22(2), 291-299. https://doi.org/10.1007/s10903-01900927-6

Bai, Y., Welk, G. J., Nam, y. H., Lee, J. A., Lee, J.-M., Kim, Y., Meier, N. F. \& Dixon, P. M. (2016). Comparison of Consumer and Research Monitors under Semistructured Settings. Medicine \& Science in Sports \& Exercise, 48(1), 151-158. http://doi.org/10.1249/ mss.0000000000000727

BC Ministry of Education (2011). Daily physical activity kindergarten to grade 12: program guide (updated 2011). British Columbia website: https://www2.gov.bc.ca/assets/gov/education/ kindergarten-to-grade-12/teach/pdfs/curriculum/ dailyphysicalactivity/program_guide.pdf

Beets, M. W., Bornstein, D., Beighle, A., Cardinal, B. J., \& Morgan, C. F. (2010). Pedometer-Measured Physical Activity Patterns of Youth. American Journal of Preventive Medicine, 38(2), 208216. http://doi.org/10.1016/j.amepre.2009.09.045

Böhm, B., Karwiese, S. D., Böhm, H., \& Oberhoffer, R. (2019). Effects of Mobile Health Including Wearable Activity Trackers to Increase Physical Activity Outcomes Among Healthy Children and Adolescents: Systematic Review. JMIR Mhealth Uhealth, 7(4), e8298. http://doi.org/10.2196/mhealth.8298

Brickwood, K.J., Watson, G., O'Brien, J., \& Williams, A.D. (2019) Consumer-Based Wearable Activity Trackers Increase Physical Activity Participation: Systematic Review and MetaAnalysis. JMIR Mhealth Uhealth, 7(4), e11819. http://doi.org/ 10.2196/11819

Bronikowski, M., Bronikowska, M., Maciaszek, J., \& Glapa, A. (2018). Maybe it is not a goal that matters: a report from a physical activity intervention in youth. The Journal of sports medicine and physical fitness, 58(3), 348-355. https://doi.org/10.23736/ S0022-4707.16.06617-1

Center for Disease Control and Prevention (CDC) (2011). How much physical activity do children neeed? CDC website: https://www. cdc.gov/physicalactivity/basics/pdfs/FrameworkGraphicV9.pdf

Chillón, P., Ortega, F. B., Ferrando, J. A., \& Casajus, J. A. (2011). Physical fitness in rural and urban children and adolescents from Spain. Journal of Science and Medicine in Sport, 14, 417-423. http:// doi.org/10.1016/j.jsams.2011.04.004

Drehlich, M., Naraine, M., Rowe, K., Lai, S. K., Salmon, J., Brown, H., Koorts, H., Macfarlane, S., \& Ridgers, N. D. (2020). Using the Technology Acceptance Model to Explore Adolescents' Perspectives on Combining Technologies for Physical Activity Promotion Within an Intervention: Usability Study. Journal of Medical Internet Research, 22(3), e15552. https://doi.org/10.2196/15552

El-Amrawy, F., \& Nounou, M. I. (2015). Are currently available wearable devices for activity tracking and heart rate monitoring accurate, precise, and medically beneficial? Healthcare Informatics Research, 21(4), 315-20. http://doi.org/10.4258/ hir.2015.21.4.315

Evenson, K. R., Goto, M. M., \& Furberg, R. D. (2015). Systematic review of the validity and reliability of consumer-wearable activity trackers. International Journal of Behavioral Nutrition and Physical Activity, 12, 159. http://doi.org/10.1186/s12966015-0314-1 
Galy, O., Yacef, K., \& Caillaud, C. (2019). Improving Pacific Adolescents' Physical Activity Toward International Recommendations: Exploratory Study of a Digital Education App Coupled With Activity Trackers. JMIR mHealth and uHealth, 7(12), e14854. https://doi.org/10.2196/14854

Garriguet, D., \& Colley, R. C. (2012). Daily patterns of physical activity among Canadians. Health reports, 23(2), 27-32.

Gaudet, J., Gallant, F., \& Bélanger, M. (2017). A Bit of Fit: Minimalist Intervention in Adolescents Based on a Physical Activity Tracker. JMIR Mhealth Uhealth, 5(7), e92. https://doi. org/10.2196/mhealth.7647

Gauthier, A. P., Laurence, M., Thirkill, L., \& Dorman, S. C. (2012). Examining School-Based Pedometer Step Counts Among Children in Grades 3 to 6 Using Different Timetables. Journal of School Health, 82(7), 311-317. http://doi.org/10.1111/j.17461561.2012.00704.x

Guthol, R., Stevens, G. A., Riley, L. M., \& Bull, F. C. (2019). Global trends in insufficient physical activity among adolescents: a pooled analysis of 298 population-based surveys with 7.6 million participants. The Lancet Child \& Adolescent Health, 4(1), 23-35. https://doi.org/10.1016/S2352-4642(19)30323-2

Hardy, L. L., Okely, A. D., Dobbins, T. A., \& Booth, M. L. (2008). Physical Activity among Adolescents in New South Wales (Australia). Medicine \& Science in Sports \& Exercise, 40(5), 835-841. http://doi.org/10.1249/mss.0b013e318163f286

Hayes, L. B., \& Van Camp, C. M. (2015). Increasing physical activity of children during school recess. Journal of Applied Behavior Analysis, 48(3), 690-695. http://doi.org/10.1002/jaba.222

Heath, G. W., Parra, D. C., Sarmiento, O. L., Andersen, L. B., Owen, N., Goenka, S., Montes, F., \& Brownson, R. C. (2012). Evidencebased intervention in physical activity: lessons from around the world. The Lancet, 38(9838), 272-281. http://doi.org/10.1016/ S0140-6736(12)60816-2

Hills, A. P., Dengel, D. R., \& Lubans, D. R. (2015). Supporting public health priorities: Recommendations for physical education and physical activity promotion in schools. Progress in Cardiovascular Diseases, 57, 368-374. http://doi. org/10.1016/j.pcad.2014.09.010

Katzmarzyk, P. T., Denstel, K. D., Beals, K., Bolling, C., Wright, C., Crouter, S.E., Mckenzie, T. L., Pate, R. R., Saelens, B. E, Staiano, A. E., Stanish, H. I., \& Sisson, S. B. (2016). Results from the United States of America's 2016 report card on physical activity for children and youth. Journal of Physical Activity and Health, 13(2), S307-S313. http://doi.org/10.1123/jpah.2016-0321

Keadle, S. K., Shiroma, E. J., Freedson, P. S., \& Lee, I.-M. (2014). Impact of accelerometer data processing decisions on the sample size, wear time and physical activity level of a large cohort study. BMC Public Health, 14(1), 1210. http://doi.org/10.1186/1471-2458-14-1210

Kim, Y., Lumpkin, A., Lochbaum, M., Stegemeier, S., \& Kitten, K. (2018). Promoting physical activity using a wearable activity tracker in college students: A cluster randomized controlled trial. Journal of Sports Sciences, 36(16), 1889-1896. http://doi. org/10.1080/02640414.2018.1423886

Kriemler, S., Meyer, U., Martin, E., Van Sluijs, E. M., Andersen, L. B., \& Martin, B. W. (2011). Effect of school-based interventions on physical activity and fitness in children and adolescents: a review of reviews and systematic update. British Journal of Sports Medicine, 45(11), 923-930. http://doi.org/10.1136/ bjsports-2011-090186

Lee, J.-M., Kim, Y., \& Welk, G. (2014). Validity of consumerbased physical activity monitors. Medicine \& Science in Sports \& Exercise, 46(9), 1840-1848. http://doi.org/10.1249/ mss.0000000000000287

Martínez-Gómez, D., Veiga, O. L., Zapatera, B., Gómez-Martínez, S., Martínez, D., \& Marcos, A. (2013). Physical Activity During High School Recess in Spanish Adolescents: The AFINOS Study. Journal of Physical Activity and Health, 11(6), 1194-1201. http://doi.org/10.1123/jpah.2012-0345
Mayorga-Vega, D., Casado-Robles, C., Viciana, J., \& LópezFernández, I. (2019). Daily Step-Based Recommendations Related to Moderate-to-Vigorous Physical Activity and Sedentary Behavior in Adolescents. Journal of Sports Science and Medicine, 18(4), 586-595.

Miqueles, J. H., Cadenas-Sánchez, C., Ekelund, U., Delisle Nyström, C., Mora-Gonzalez, J., Löf, M., Labayen, I., Ruiz, J. R., \& Ortega, F. B. (2017). Accelerometer Data Collection and Processing Criteria to Assess Physical Activity and Other Outcomes: A Systematic Review and Practical Considerations. Sports Medicine, 47(9), 1821-1845. http://doi.org/10.1007/s40279017-0716-0

Miguel-Berges, M., Santaliestra-Pasias, A., Mouratidou, T., De Miguel-Etayo, P., Androutsos, O., De Craemer, M., Galcheva, S., Koletzko, B., Kulaga, Z., Manios, Y., \& Moreno, L. on behalf of the ToyBox-study group. (2019). Combined Longitudinal Effect of Physical Activity and Screen Time on Food and Beverage Consumption in European Preschool Children: The ToyBox-Study. Nutrients, 11(5), 1048. http://doi.org/10.3390/ nu17051048

Nanney, L. (2014). Self-determination theory and movement technology in college physical activity classes (Master's Thesis, East Carolina University). http://hdl. handle.net/10342/4576.

Otzen, T., \& Manterola, C. (2017). Sampling techniques on a population study. International Journal of Morphology, 35(1), 227-232.

Pearson, E.S. (2012). Goal setting as health behavior change strategy in overweight and obese adults: A systematic literature review examining intervention components. Patient Education and Counseling, 87, 32-42. http://doi.org/10.1016/j. pec.2011.07.018

Pittman, A. F. (2018). Effect of a School-Based Activity Tracker, Companion Social Website, and Text Messaging Intervention on Exercise, Fitness, and Physical Activity Self-Efficacy of Middle School Students. The Journal of School Nursing, 36(2), 112-120. http://doi.org/10.1177/1059840518791223

Price, K., Bird, S. R., Lythgo, N., Raj, I. S., Wong, J. Y. L., \& Lynch, C. (2017). Validation of the Fitbit One, Garmin Vivofit and Jawbone UP activity tracker in estimation of energy expenditure during treadmill walking and running. Journal of Medical Engineering \& Technology, 41(3), 208-215. http://doi.org/10.1080/030919 02.2016 .1253795

Ridgers, N. D., McNarry, M. A., \& Mackintosh, K. A. (2016). Feasibility and effectiveness of using wearable activity trackers in youth: a systematic review. JMIR Mhealth Uhealth, 4(4), e129. http:// doi.org/10.2196/mhealth. 6540

Rote, A. E. (2016). Physical activity intervention using Fitbits in an introductory college health course. Health Education Journal, 76(3), 337-348. http://doi.org/10.1177/0017896916674505

Santaliestra-Pasías, A. M., Dios, J. E. L., Sprengeler, O., Hebestreit, A., De Henauw, S., Eiben, G., Felso, R., Lauria, F., Tornaritis, M., Veidebaum, T., Pala, V., \& Moreno, L. A. (2018). Food and beverage intakes according to physical activity levels in European children: the IDEFICS (Identification and prevention of Dietary and lifestyle induced health EFfects In Children and infantS) study. Public Health Nutrition, 21(09), 1717-1725. http://doi.org/10.1017/s1368980018000046

Sperlich, B., \& Holmberg, H.-C. (2017). Wearable, yes, but able...?: it is time for evidence-based marketing claims! British Journal of Sports Medicine, 51(16), 1240-1240. http://doi.org/10.1136/ bjsports-2016-097295

Tremblay, M. S., Aubert, S., Barnes, J. D., Saunders, T. J., Carson, V., Latimer-Cheung, A. E., Chastin, S. F. M., Altenburg, T. M., Chinapaw, M. J. M., \& on behalf of SBRN Terminology Consensus Proyect Participants. (2017). Sedentary Behavior Research Network (SBRN) - Terminology Consensus Project process and outcome. International Journal of Behavioral Nutrition and Physical Activity. 14, 75. http://doi.org/ 10.1186/ s12966-017-0525-8 
Tudor-Locke, C., McClain, J. J., Hart, T. L., Sisson, S. B., \& Washington, T. L. (2009). Expected Values for PedometerDetermined Physical Activity in Youth. Research Quarterly for Exercise and Sport, 80(2), 164-174. http://doi.org/10.1080/02 701367.2009.10599550

Tudor-Locke, C., Craig, C. L., Beets, M. W., Belton, S., Cardon, G. M., Duncan, S., Hatano, Y., Lubans, D. R., Olds, T. S., Raustorp, A., Rowe, D. A., Spence, J. C., Tanaka, S. \& Blair, S. N. (2011). How many steps/day are enough? for children and adolescents. International Journal of Behavioral Nutrition and Physical Activity, 8 , (78), 1-14. https://doi.org/10.1186/1479-5868-8-78

U.S. Department of Health and Human Services (2018). Physical Activity Guidelines for Americans, 2nd edition. U.S. website: https://health.gov/sites/default/files/2019-09/Physical_ Activity_Guidelines_2nd_edition.pdf

Van Tuyckom, C., \& Scheerder, J. (2010). A multilevel analysis of social stratification patterns of leisure-time physical activity among Europeans. Science \& Sports, 25(6), 304-311. http:// doi.org/10.1016/j.scispo.2010.04.003

Wang, L., Liu, T., Wang, Y., Li, Q., Yi, J., \& Inoue, Y. (2017). Evaluation on Step Counting Performance of Wristband Activity Monitors in Daily Living Environment. IEEE Access, 5, 1302013027. http://doi.org/10.1109/access.2017.2721098
Wijtzes, A.I., Verloigne, M., Mouton, A., Cloes, M., De Ridder, K.A. Cardon, G., \& Seghers, J. (2016). Results from Belgium's 2016 report card on physical activity for children and youth. Journal of Physical Activity and Health, 13(2), S95-S103. http://doi. org/10.1123/jpah.2016-0306

World Health Organization (WHO) (2010). Global recommendations on physical activity for health. WHO website: https://apps.who. int/iris/bitstream/handle/10665/44441/9789243599977_spa. pdf

Wyszyńska, J., Matłosz, P., Szybisty, A., Lenik, P., Dereń, K., Mazur, A., \& Herbert, J. (2020). Obesity and Body Composition in Preschool Children with Different Levels of Actigraphy-Derived Physical Activity-A Cross-Sectional Study. Journal of Clinical Medicine, 9(4), 1210. https://doi.org/10.3390/jcm9041210

Zongo, P., Frayon, S., Antoine-Jonville, S., Wattelez, G., Le Roux, P.Y., Hue, O., \& Galy, O. (2017). Anthropometric Characteristics and Physical Fitness in Rural and Urban 11- to 16-Year-Old Melanesian Adolescents: A Cross-sectional Study in New Caledonian Schools. Asia Pacific Journal of Public Health, 29(7), 589-598. https://doi.org/10.1177/1010539517735414 